Revue d'histoire de l'enfance « irrégulière »

Le Temps de l'histoire

15 | 2013

Enfances déplacées. (II) en temps de guerre

\title{
Pieds nus dans les ruines : le regard de Chim sur les enfants de la Guerre
}

À propos de l'ouvrage : Chim : Children of War de Carole Naggar

David Niget

\section{OpenEdition}

Journals

Édition électronique

URL : http://journals.openedition.org/rhei/3504

DOI : $10.4000 /$ rhei.3504

ISSN : $1777-540 \mathrm{X}$

Éditeur

Presses universitaires de Rennes

Édition imprimée

Date de publication : 30 octobre 2013

Pagination : 143-145, I-IV

ISBN : 978-2-7535-2896-3

ISSN : $1287-2431$

Référence électronique

David Niget, «Pieds nus dans les ruines : le regard de Chim sur les enfants de la Guerre », Revue

d'histoire de l'enfance « irrégulière » [En ligne], 15 | 2013, mis en ligne le 30 octobre 2013, consulté le 22 septembre 2020. URL : http://journals.openedition.org/rhei/3504 ; DOI : https://doi.org/10.4000/rhei. 3504

Ce document a été généré automatiquement le 22 septembre 2020

(C) PUR 


\title{
Pieds nus dans les ruines : le regard de Chim sur les enfants de la Guerre
}

\author{
À propos de l'ouvrage : Chim : Children of War de Carole Naggar
}

\section{David Niget}

\section{RÉFÉRENCE}

Chim : Children of War, 2013, New York, Umbrage Editions, 2013, ISBN 9781884167836

1 Chim, alias David Seymour, compagnon de route de Robert Capa et de Gerda Taro, a vu les enfants de la guerre. Dès l'équipée espagnole, il prête une attention particulière aux petits, victimes iconiques des guerres civiles $d u x^{e}$ siècle ${ }^{1}$. Chim est du côté des enfants, comme en témoigne sa «lettre à un adulte», douce et révoltée, qui accompagne l'album réalisé pour l'UNICEF dans les ruines de l'Europe, en 1948. La première rétrospective qui lui est consacrée, à Chicago, en 1957, au lendemain de sa mort accidentelle, s'intitule Chim's Children; ce sont «ses » enfants. Chim incarne avec force la photographie humaniste qui a marqué la naissance du photoreportage au $\mathrm{xx}^{\mathrm{e}}$ siècle, cette photographie qui s'est penchée sur l'enfance et les enfants, comme symboles mais aussi comme sujets.

2 Un ouvrage, publié par Carole Naggar, photographe et historienne, est dédié à un aspect fondateur du regard de Chim, l'attention portée à l'enfance tourmentée par la guerre. Si l'on y trouve des clichés célèbres, dont cette image de Terezka, jeune fille au regard d'épouvante traçant à la craie un entrelacs tortueux censé représenter sa maison, Carole Naggar, nous présente nombre d'images inédites tirées de ce voyage européen de 1948. Ces images, de grande qualité, sont replacées dans leur contexte, commentées avec la justesse de l'œil photographique, et accompagnées d'une introduction biographique et d'annexes précieuses.

3 Chim, né Dawid Szymin à Varsovie en 1911 dans une famille d'intellectuels juifs, a étudié l'art à Leipzig. Au début des années trente, il émigre à Paris, poussé par la dégradation de la situation économique et politique en Allemagne et en Pologne. Il y 
rencontre Cartier-Bresson et Capa, dans l'engagement socialiste qui saisit cette génération d'artistes. Il fait ses armes de photographe sous le Front populaire, dont il dépeint les grandes grèves pour le magazine Regards. Son militantisme le pousse naturellement vers l'Espagne de la guerre civile, où il découvre les atrocités de la guerre moderne, et spécialement le sort réservé aux enfants, sur lesquels, déjà, il pose un regard empathique. Il suivra les réfugiés de la guerre au-delà des Pyrénées, et jusqu'au Mexique, à la fin des années 1930.

4 Après avoir cofondé, en 1947, l'agence Magnum, il est sollicité par l'UNESCO pour rendre compte du travail de l'UNICEF auprès des 13 millions d'enfants réfugiés d'Europe. Il prend sa tâche à cœur, et les quelques semaines de mission deviennent des mois de travail attentif, au plus près des enfants de Grèce, d'Italie, de Hongrie, d'Autriche, d'Allemagne, et de Pologne. L'opuscule, Children of Europe, paraît en 1949, et connaît une diffusion mondiale, faisant de Chim «le photographe des enfants ». Son travail s'inscrit dans une œuvre graphique contemporaine attentive au sort des jeunes victimes de la guerre, faisant écho aux images de Werner Bischof, de Thérèse Bonney ou de Denise Bellon. On pense également au Sciuscià de Vittorio De Sica (1946), à Allemagne, année zéro, de Roberto Rossellini (1948) ou encore aux Enfants d'Hiroshima, de Shindo Kaneto (1952). L'enfant des ruines est une figure importante de la culture pacifiste issue de la guerre.

5 De manière très neuve, Chim adopte le point de vue des enfants. Il est bien accepté par les jeunes avec qui il noue des liens, et porte sur eux un regard compréhensif sans être larmoyant. De ses images, on lit la joie de l'enfance qui affleure malgré la souffrance, la gravité de l'enfance face à l'absurde monde. Cette posture permet à Chim de reconnaître la violence infligée aux plus jeunes lors des conflits visant de plus en plus de civils, mais sans dénaturer l'identité enfantine. Pourtant, au sortir de la guerre, certains stéréotypes circulent, tels que celui des enfants-loups retournés à l'état sauvage. Un discours psychiatrique porte sur les « enfants perdus ", jeunes abandonnés et déportés, enfants-monstres ayant perdu leur enfance, vieillis trop vite. Chim introduit là la complexité du regard humaniste, décrivant des enfants affligés par les conflits, souvent handicapés, désocialisés, délinquants, mais vivants, joueurs, avides d'avenir. Le regard de Chim est compatissant mais il est aussi moral. La déviance y est omniprésente - jeunes prostituées, jeunes mendiants et délinquants; et si elle n'est pas condamnée comme telle, elle est posée comme le signe d'un échec du monde des adultes. La question de l'errance y est récurrente; on est témoin de ces processions d'écoliers dans les plaines de ruines polonaises et allemandes, de ces évacuations navales dantesques, dans une Grèce écartelée par la guerre civile, de ces enfants refugiés dormant dans des hamacs ou en quinconce dans des lits étriqués, de ces chaussures percées portant les stigmates de l'exil et de ces petits pieds nus sur les gravats.

6 Regard libre, ce travail de commande s'inscrit néanmoins dans une entreprise de propagande, qui vise, en premier lieu, à collecter des fonds pour les enfants d'Europe auprès des donateurs américains, et à promouvoir, indirectement, le Plan Marshall. Jamais on ne mentionne, d'ailleurs, que ces enfants ont été bombardés par les Alliés eux-mêmes. Le reportage de 1948 est aussi empreint du climat d'entrée dans la guerre froide, alors que les vainqueurs occidentaux cherchent à promouvoir la démocratie, dont les enfants sont « les graines du destin ». Ce slogan évocateur est le titre du film réalisé en 1946 par l'armée américaine pour louer les travaux de l'UNRRA 
(Administration des Nations unies pour les secours et la reconstruction), dont le travail de Chim pour l'UNICEF est la suite. Ces enfants incarnent la promesse d'un avenir pacifié, libéral, et industrieux. Fleurs des ruines, ces images d'enfants doivent transcender les clivages politiques pour atteindre cet idéal qui masque mal les conflits latents à venir, dans un monde bipolaire déchiré par les guerres de décolonisation dont les enfants les premières victimes, jusqu'à ce que la figure de l'enfant-soldat vienne, subrepticement, remplacer celle du martyr. 
7 Mais ces images n'appartiennent pas aux propagandistes, elles disent aussi, avec ferveur, la fragilité et la force de l'enfance dans les tourments du siècle, et nous parviennent à travers l'histoire comme une voix d'enfant après le vacarme des bombes.

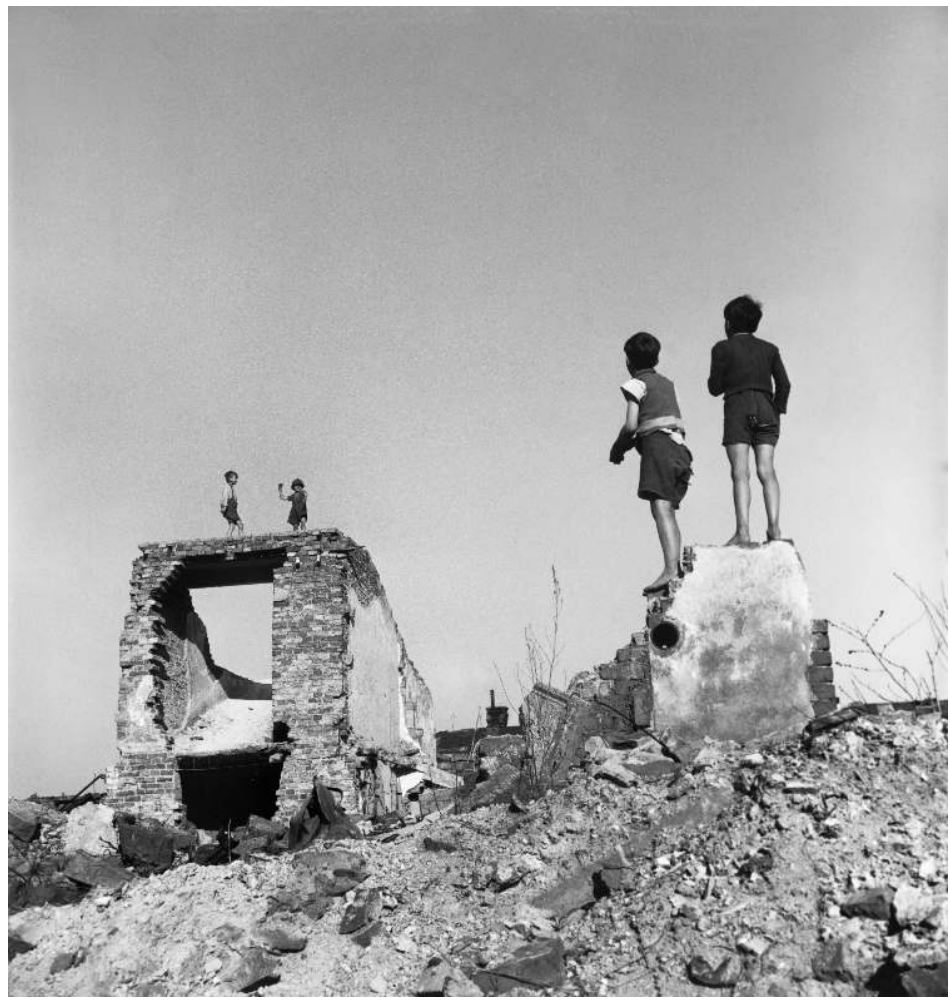

Autriche, 1948. Des garçons jouent dans les immeubles bombardés du quartier populaire de Favoriten. L'ambiance de cette image évoque celle des clichés de Cartier Bresson. @ David Seymour/ Magnum et Carole Naggar, courtesy Umbrage Editions.

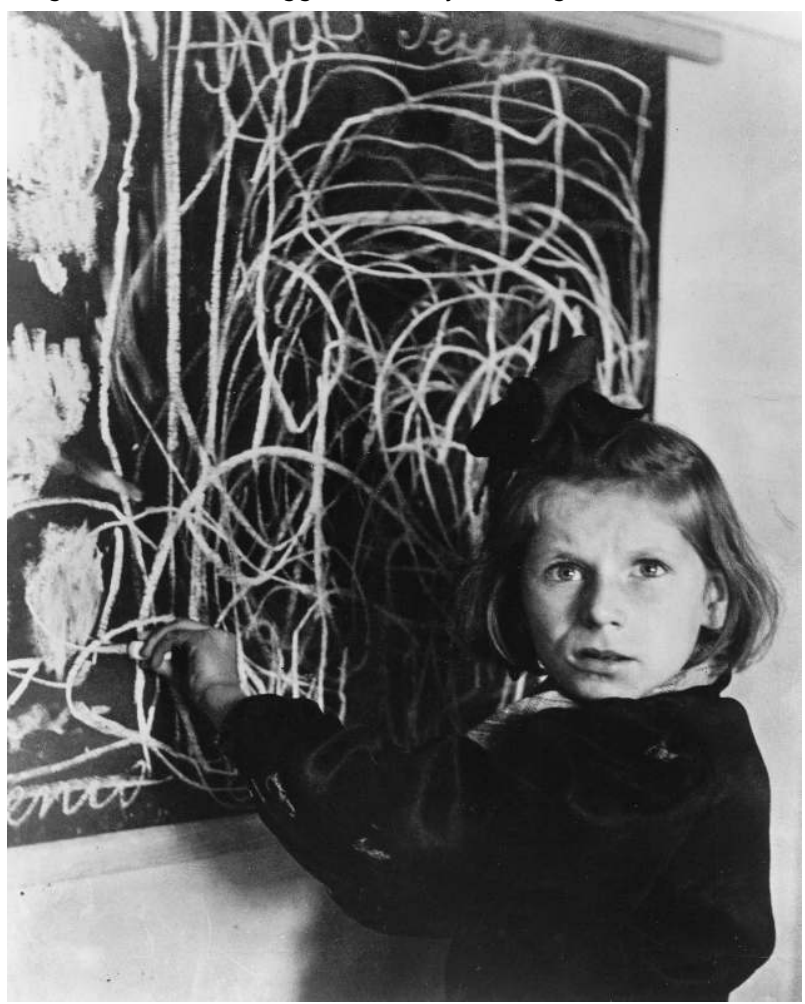


Pologne, 1948. Terezka est une élève d'une institution polonaise pour enfants perturbés. Elle fait ces gribouillages lorsqu'on lui demande de dessiner sa maison. Le portrait de Terezka, un des plus connus de Chim, a été utilisé comme couverture de livres consacrés à la peur, au traumatisme et à

l'Holocauste; c'est aussi l'emblème de la fondation allemande éponyme. (c) David Seymour/Magnum et Carole Naggar, courtesy Umbrage Editions.

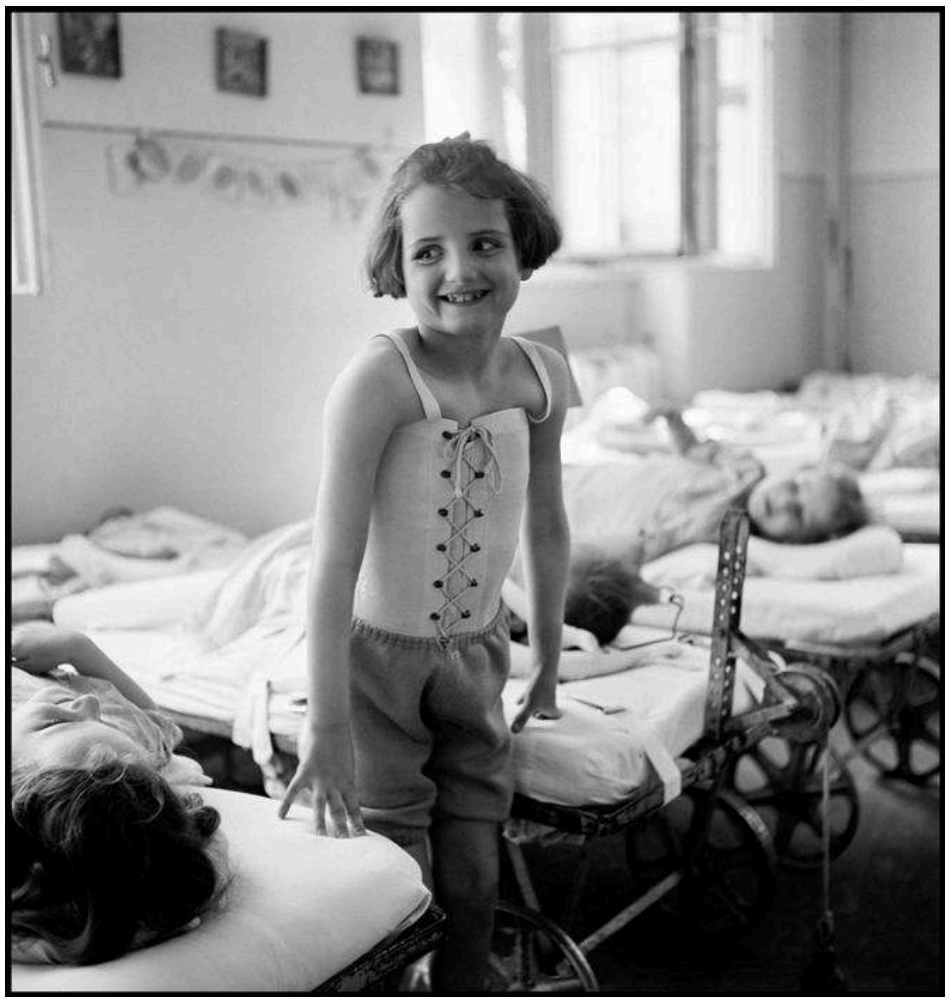

Autriche, Vienne, 1948. L'hôpital pour enfants de Bellevue. Cette fillette, souffrant de tuberculose vertébrale, doit porter un corset rigide. Chim a laissé flous la chambre d'hôpital et les enfants à l'arrière-plan, portant son attention sur la jeune fille, son sourire et le léger toucher de ses doigts sur le lit. @ David Seymour/Magnum et Carole Naggar, courtesy Umbrage Editions. 


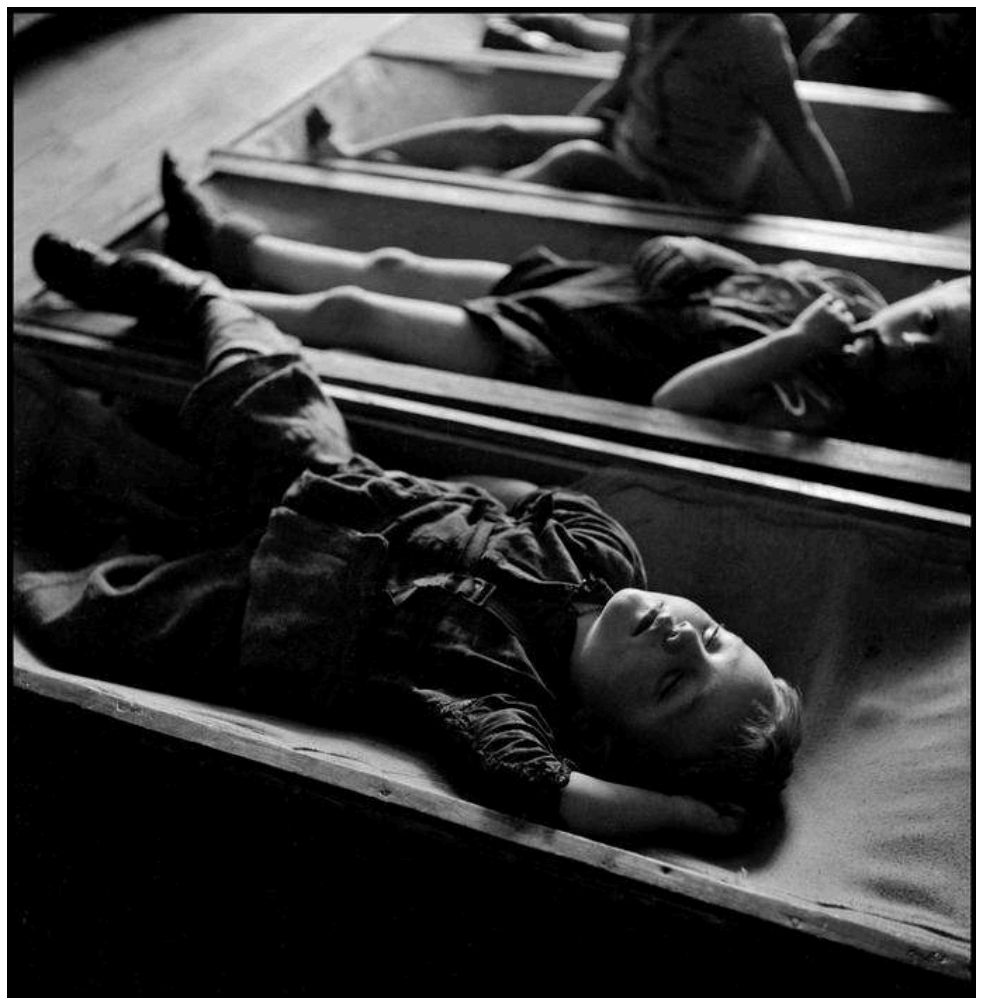

Autriche, Vienne, 1948. Un vieil arsenal à moitié détruit est devenu le camp de réfugiés des Sudètes. Ils vivent ici et travaillent dans les fermes et l'industrie autrichienne. La nurserie est dirigée par les réfugiés eux-mêmes. Quatre-cent-mille enfants sont alors nourris par I'UNICEF en Autriche. Tous ont en dessous de six ans. (C) David Seymour/Magnum et Carole Naggar, courtesy Umbrage Editions. 


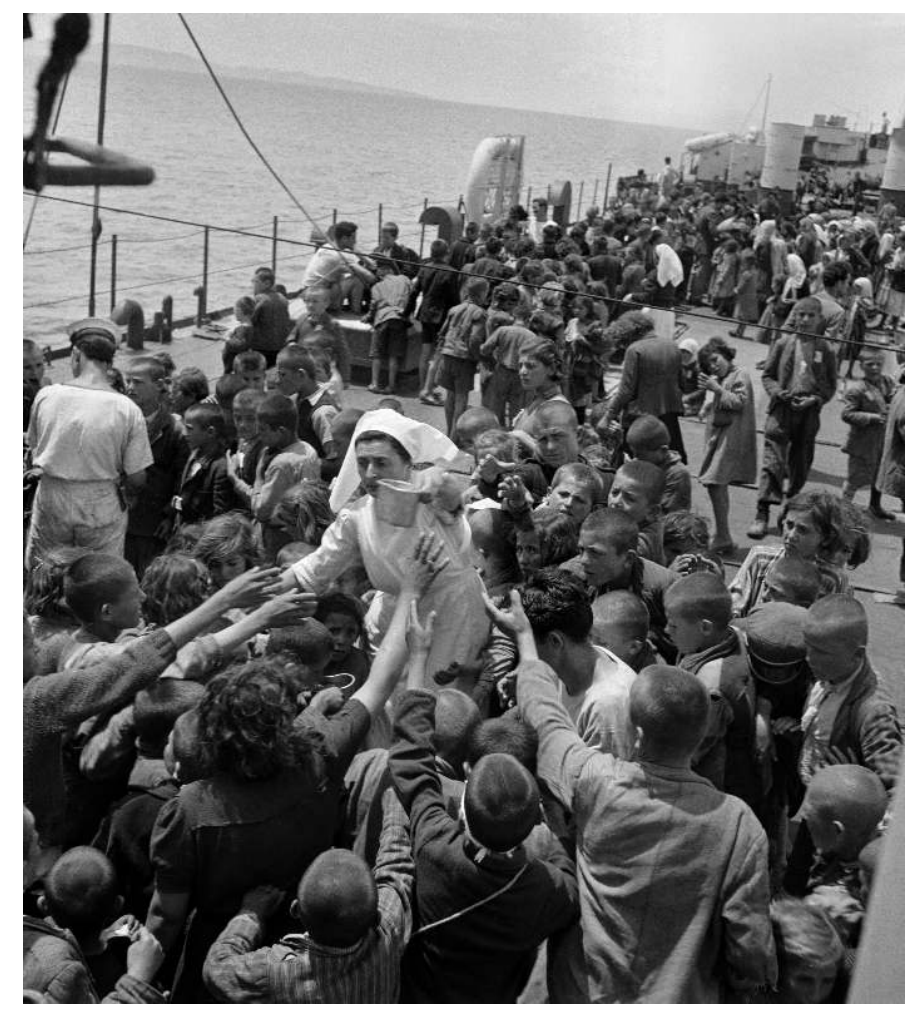

Grèce, 1948. À bord du Samos, le navire qui a évacué les enfants des zones de guerre civile. Les bras tendus des enfants convergeant vers la religieuse, au centre, sont caractéristiques de la théâtralité et du sens de la composition de Chim, s'adressant au spectateur. Notre regard s'égare ensuite, suivant une ligne oblique vers l'horizon, symbolisant l'inconnu vers lequel ces enfants sont conduits. (c) David Seymour/Magnum et Carole Naggar, courtesy Umbrage Editions. 


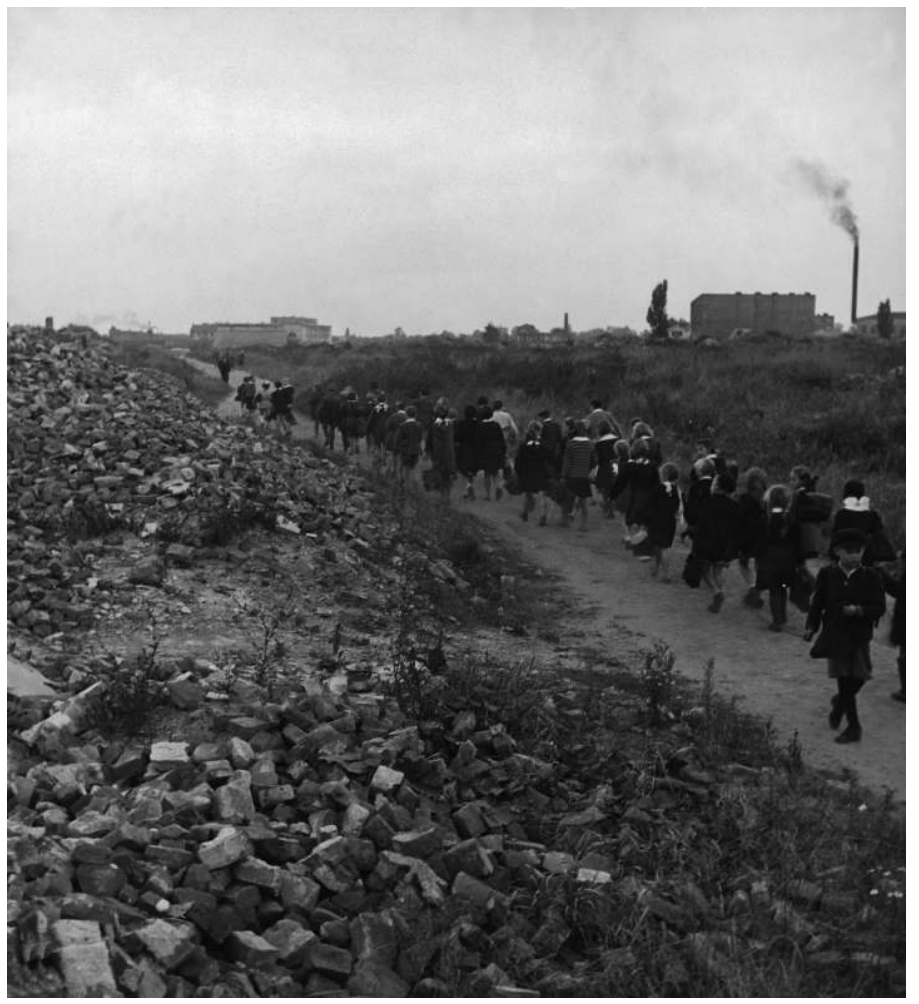

Pologne, Varsovie, 1948. Des écoliers quittant leur école secondaire située aux marges du ghetto juif, ayant ainsi échappé à la destruction. Cette image évoque une série de clichés réalisée par son confrère, Georges Rodger, à Londres durant le Blitz. @ David Seymour/Magnum et Carole Naggar, courtesy Umbrage Editions.

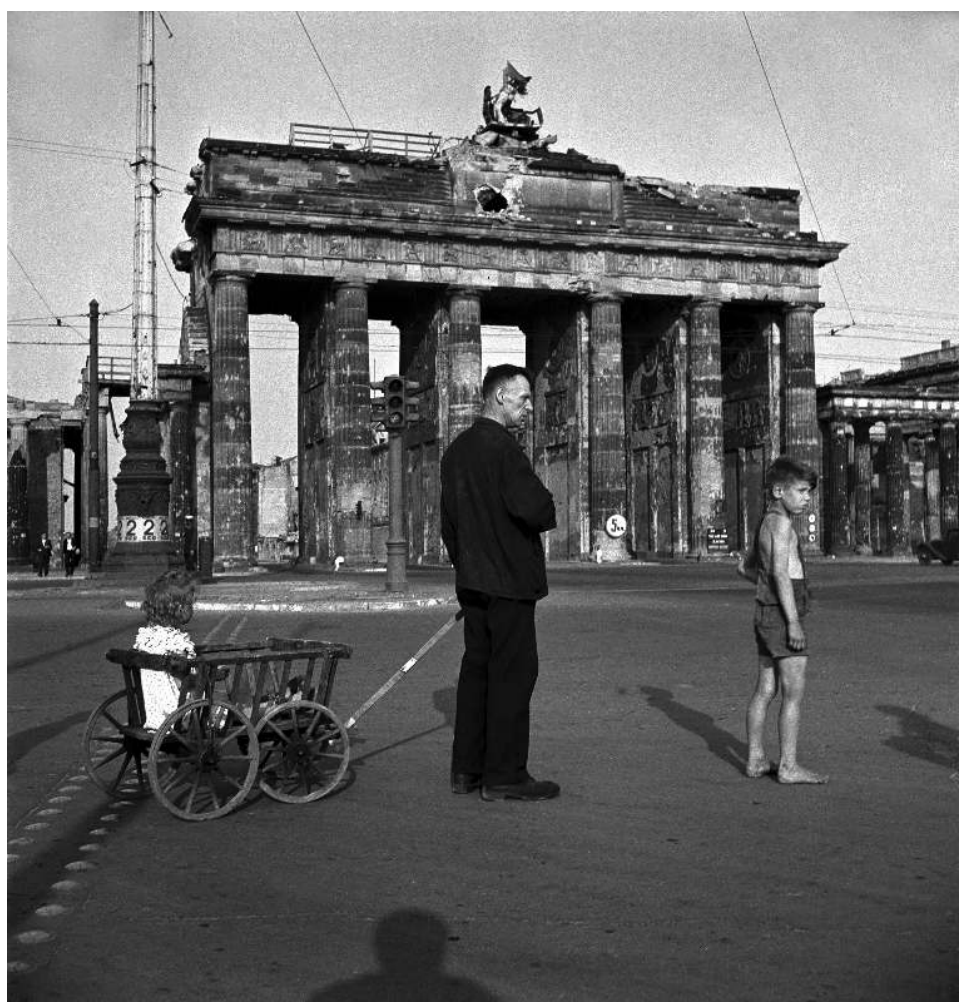

Allemagne, Berlin, 1947. Un homme marche avec ses enfants à proximité de la porte de Brandebourg. Ce monument, symbole de paix, est surmonté d'un quadrige conduit par un ange ailé, alors détruit. L'ombre du photographe est visible au bas de l'image. (c) David Seymour/Magnum et Carole Naggar, courtesy Umbrage Editions. 


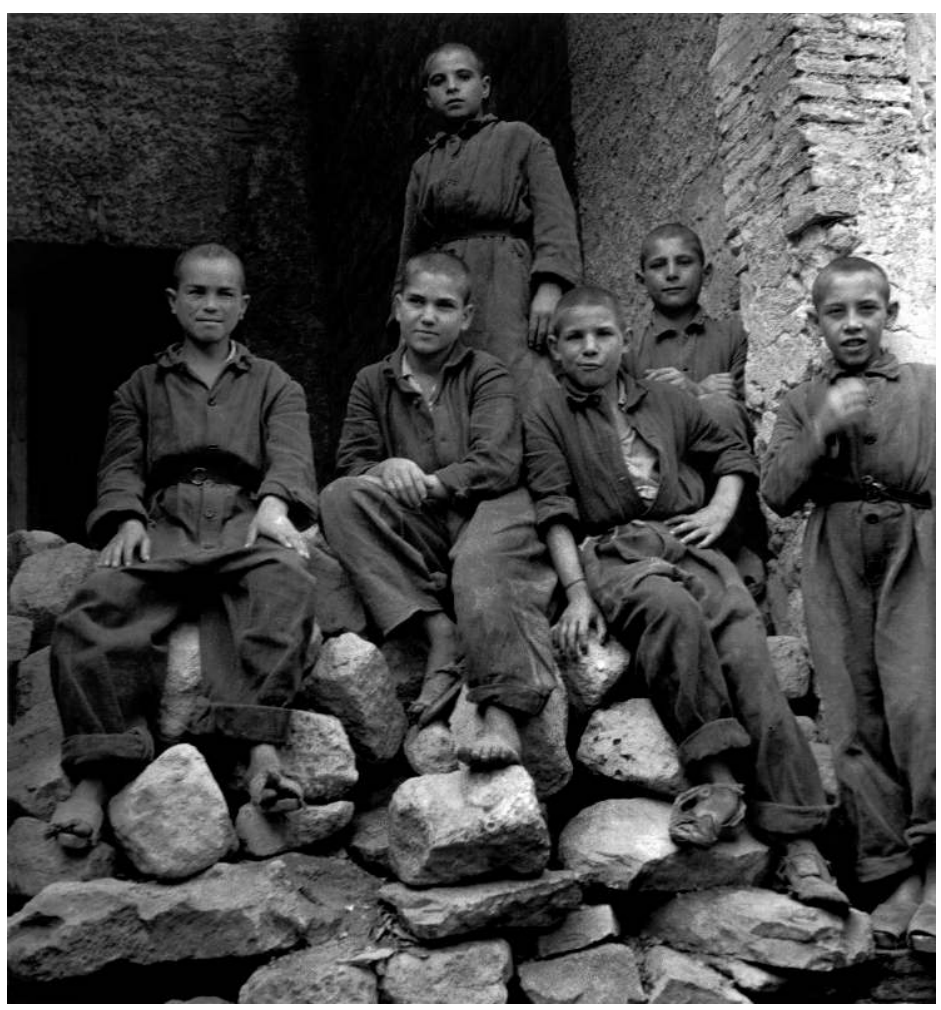

Italie, Naples, 1948. Une école de réforme pour garçons à l'Albergo dei Poveri, où les enfants sont placés par la cour des jeunes délinquants. Les garçons posent avec malice, comme pour une photo de famille, le visage tantôt triste, suspicieux ou grimaçant. (C) David Seymour/Magnum et Carole Naggar, courtesy Umbrage Editions.

\section{NOTES}

1. Young Cynthia et MATTHIEUSSENT Brice, La valise mexicaine: Capa, Chim, Taro: les négatifs retrouvés de la guerre civile espagnole. [Exposition, Arles, Musée départemental de l'Arles antique, 4 juillet-18 septembre 2011], Arles, Actes Sud, 2011.

\section{AUTEURS}

\section{DAVID NIGET}

David Niget est maître de conférences à l'université d'Angers, membre du laboratoire CERHIO 